\title{
SENTIDOS ASSOCIADOS À VIOLÊNCIA PARA IDOSOS E PROFISSIONAIS
}

\author{
Meanings associated with violence for elderly and professionals \\ Significados asociados con la violencia a las personas mayores y profesionales
}

Tatyanni Peixoto Rodrigues ${ }^{1}$

Adriana de Azevedo F. Smith ${ }^{4}$

\author{
Maria Adelaide Silva P. Moreira² \\ Jose Luiz Telles de Almeida ${ }^{5}$
}

Antonia Oliveira Silva ${ }^{3}$

Manuel José Lopes ${ }^{6}$

\section{RESUMO}

Objetivos: Conhecer os sentidos associados à violência segundo os idosos e os profissionais. Métodos: Pesquisa exploratória com abordagem qualitativa, realizada no serviço de curadoria do cidadão/delegacia especializada, na cidade de João Pessoa-PB, através de uma entrevista semiestruturada com 30 idosos e 4 profissionais que atendem o idoso vitimado. Os dados obtidos das entrevistas foram processados pelo programa informático Alceste 4.8. Resultados: Os dados foram constituídos por um corpus correspondente a 34 entrevistas, com $86,87 \%$ de aproveitamento, apontando para duas grandes classes: sentidos atribuídos a violência pelos idosos; profissionais e impacto da violência na vida do idoso segundo os sujeitos entrevistados. Conclusão: Os maus-tratos e a negligência contra os idosos constituem um grave problema, ainda não diagnosticado em toda a sua extensão, sobretudo no âmbito familiar e institucional, merecendo, portanto, maior atenção de órgãos governamentais e não governamentais.

Palavras-chave: Violência. Saúde. Idoso. Enfermagem.

\begin{abstract}
Objectives: Apprehend the social representations of violence made by older people and professionals that serve the elderly at the complaints. Methodology: This is an exploratory research using a qualitative approach, conducted with the guardianship of the public service/office specialist in the city of João Pessoa- Paraíba, with thirty-four seniors who attend the elderly victim, who answered a semi-structured interview. Data were processed by Alceste 4.8 software. Results: The data submitted to Alceste 4.8 software, was formed by a corpus corresponding to 34 units of text or Initial Context (ICU's), withdrew from the hierarchical analysis 105 words, equivalent to $86.87 \%$, pointing to two major classes: meanings attributed to violence and the elderly and professional impact of violence on the lives of the elderly according to the subjects interviewed. Final considerations: the social representations about the consequences of violence to the elderly and their impact on the professional front on this issue indicate that the studies so far carried out in Brazil on violence considers this phenomenon a public health problem. The ill-treatment and neglect against the elderly are a serious problem, not yet diagnosed in all its extension, especially in the family and institutional, deserving, therefore greater attention from government and non-governmental organizations.
\end{abstract}

Key-words: Violence. Health. Aging. Nursing.

\section{Resumen}

Objetivos: conocer los sentidos asociados a la violencia según los ancianos y los profesionales. Metodología: Se trata de una investigación exploratoria, utilizando un enfoque cualitativo, realizado con la tutela de la administración pública y especialista en la ciudad de João Pessoa- Paraíba con treinta ancianos y cuatro profesionales que asisten las víctimas de edad avanzada, que responde a una entrevista seme-estructurada. Los datos fueron procesados por el software Alceste 4.8. Resultados: Los datos fueron creados por el corpus correspondiente a 34 entrevistas, con 86,87\% de recuperación, apuntando a dos clases principales: los significados atribuidos a la violencia y los ancianos y el impacto profesional de la violencia en la vida a los ancianos de acuerdo a los entrevistados. Consideraciones finales: las representaciones sociales acerca de las consecuencias de la violencia a los ancianos y su impacto frente al profesional sobre este tema indican que los estudios hasta ahora realizados en Brasil sobre la violencia considera este fenómeno un problema de salud pública. Los malos tratos y la desatención de los ancianos son un problema serio, aún no se diagnostica en toda su extensión, especialmente en el ámbito familiar e institucional, que merece, por lo tanto una mayor atención del gobierno y de organizaciones no gubernamentales.

Palabras clave: Violencia. Salud. Ancianos. Enfermería.

'Enfermeira. Participante do Grupo Internacional de Estudos e Pesquisas sobre Envelhecimento e Representações Sociais - GIEPERS da Universidade Federal da Paraiba. João PessoaPB. Brasil.E-mail: tatchy.rodrigues@gmail.com, ,Fisioterapeuta. Professor do Programa de Pós-Graduação em Saúde e Enfermagem da Universidade Estadual do Sudoeste da Bahia. Pesquisadora Colaboradora do CITS, Universidade de Évora, PT.Pesquisadora do Grupo Internacional de Estudos e Pesquisas sobre Envelhecimento e Representações Sociais -

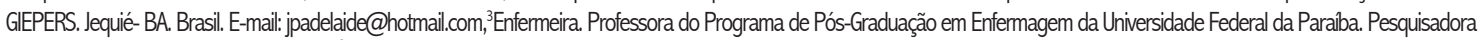
Colaboradora do CITS, Universidade de Évora, PT.Pesquisadora do CNPq. Líder do Grupo Internacional de Estudos e Pesquisas sobre Envelhecimento e Representações Sociais GIEPERS da Universidade Federal da Paraiba. João Pessoa-PB. Brasil. E-mail: alfaleda@hotmail.com, ${ }^{4}$ Mestranda do Programa de Pós-Graduação em Enfermagem da Universidade Federal da Paraiba. Participante do Grupo Internacional de Estudos e Pesquisas sobre Envelhecimento e Representações Sociais - GIEPERS da Universidade Federal da Paraiba. João PessoaPB. Brasil. E-mail: drikasmith@hotmail.com,5Médico. Professor da Escola Nacional de Saúde Pública - Fiocruz. Pesquisador do Grupo Internacional de Estudos e Pesquisas sobre Envelhecimento e Representações Sociais - GIEPERS da Universidade Federal da Paraiba; Ministério da Saúde. Brasilia-DF. Brasil. E-mail: telles@ensp.fiocruz.br, EEnfermeiro. Professor Coordenador da Universidade de Évora, PT. Pesquisador do Grupo Internacional de Estudos e Pesquisas sobre Envelhecimento e Representações Sociais - GIEPERS.Lisboa, Portugal. E-mail: mil@uevora.pt 


\section{INTRODUÇÃO}

0 envelhecimento populacional torna-se um desafio tanto nos países desenvolvidos quanto nos países em desenvolvimento. Sabe-se que é uma pretensão de qualquer sociedade, mas não basta por si só. É necessário que esse processo venha acompanhado de qualidade de vida e respeito à população idosa.'

0 aumento acelerado e abrupto do número de idosos na população brasileira provoca impacto nas políticas públicas nacionais. ${ }^{2} \mathrm{~A}$ implantação efetiva das políticas específicas para a população idosa, como a Política Nacional de Atenção à pessoa idosa e particularmente a Política Nacional de Saúde da Pessoa Idosa (PNSPI), torna-se necessária e deve ser foco de atenção para que o idoso viva mais, mas principalmente com dignidade, e que tenha seus direitos respeitados. Além disso, torna-se necessário o conhecimento dos profissionais de saúde sobre o processo de envelhecimento, pois eles devem se tornar hábeis para lidar com essa clientela. ${ }^{3,4}$

Associado a esse envelhecimento populacional, constata-se o surgimento de alguns fenômenos cada vez mais comuns, como os maus-tratos. A declaração de Toronto assinada pelos países membros da ONU em 2002 define "maus tratos ao idoso" como: qualquer ato isolado ou repetido, ou a ausência de ação apropriada, que ocorre em qualquer relacionamento em que haja uma expectativa de confiança, e que cause dano, ou incômodo a uma pessoa idosa. ${ }^{5} \mathrm{E}$ esses eventos tornam-se progressivamente frequentes, em decorrência das desigualdades sociais, com consequências desastrosas, como o aumento da violência, da pobreza e da miséria. Essa violência vem se transformando em um fator negativo para a sociedade e é um dos principais desafios para a saúde pública. ${ }^{6,7}$

Segundo definição da Organização Mundial da Saúde OMS, as violências são caracterizadas pelo uso intencional da força física ou do poder, real ou em ameaça, con-tra si próprio, contra outra pessoa, ou contra um grupo ou comunidade que possa resultar em ou tenha alta probabilidade de resultar em morte, lesão, dano psicológico, problemas de desenvolvimento ou privação.

Para Minayo e Coimbra Júnior, ${ }^{8}$ a violência contra os idosos não ocorre só no Brasil: faz parte da violência social em geral e constitui um fenômeno universal. A problemática da violência não é recente. Desde o fim da década de 1990, tem sido estudada e evidenciada das mais diversas formas. 0 Centro Latino-Americano de Estudos sobre Violência e Saúde (CLAVES) da Fundação Oswaldo Cruz (Fiocruz) vem realizando estudos e pesquisas salientando entre 1980 e 1998, houve um aumento das mortes por violência com pessoas idosas. Esse fato demonstra que na década de 80 estas mortes violentas passaram a representar uma das principais causas de mortalidade em idosos no país, assumindo em 1989, o segundo lugar, perdendo apenas para as doenças do aparelho circulatório.

A problemática dos maus-tratos contra a pessoa idosa é um problema cada vez mais grave e comum. Estes têm sido vítimas dos mais diferentes tipos de violência que vão desde insultos e espancamentos pelos próprios familiares e cuidadores até os maus-tratos sofridos em transportes públicos, instituições públicas e privadas que atendem esta população. ${ }^{7}$

Compreender a subjetividade envolvida na violência contra pessoas idosas pode apontar pistas importantes para um atendimento mais integral a essa população, considerando não só o ato em si, mas todo um conjunto simbólico associado aos diferentes tipos de abusos. ${ }^{7}$

A teoria das representações sociais, proposta por Moscovici ${ }^{9}$ enquanto uma possibilidade de conhecimento de teorias do senso comum, possibilita não apenas apreender representações como um sistema de cognições, mas destacar o contexto no qual elas são produzidas. A compreensão dessa realidade comum no grupo social a ser estudado configura-se uma via de apreensão de uma realidade social singular que poderá servir de base para a realização de futuras estratégias de ação que visem à melhoria da implementação da política institucional eficazes para o grupo estudado. Vale salientar que essas representações intervêm o processo de cognição dos indivíduos a par de fatores como o sistema de valores, fatores afetivos, imagens e motivações internas. ${ }^{10}$

Neste sentido, este estudo tem o objetivo de conhecer os sentidos associados a violência segundo os idosos e os profissionais do serviço de curadoria do cidadão/delegacia especializada que atendem ao idoso vitimado.

\section{METODOLOGIA}

Trata-se de uma pesquisa exploratória com uma abordagem qualitativa, que explorou as falas dos sujeitos, suas experiências e pertença grupal, realizada no Serviço de Curadoria do Cidadão/Delegacia Especializada, na cidade de João Pessoa, Paraíba, Brasil.

Constituídos por amostragem de conveniência, participaram deste estudo 30 idosos, com 60 anos, durante os meses de maio e junho de 2009, em condiç̃̃es de responder a entrevista; e quatro profissionais do serviço de curadoria do cidadão/delegacia especializada que atendem o idoso vitimado, correspondendo o número total de profissionais do referido serviço. Todos responderam a entrevista semiestruturada única, que contemplou duas partes: a primeira constituiu-se de teste da associação livre de palavras, com os termos indutores: "violência» e «iviolência em idososı"; e a segunda parte agrupou questões que contemplam a teoria das representações sociais centradas na violência eviolência em idosos como questões norteadoras do estudo. Todos assinaram o termo 
de Consentimento Livre e Esclarecido, conforme preconiza a Resolução 196/96 CNS/MS, após aprovação pelo Comitê de Ética do Centro de Ciências daSaúde da Universidade Federal da Paraiba, com o protocolo n. ${ }^{5}$ 53/2009.

Os dados obtidos tanto do teste da associação livre de palavras quanto da entrevista foram processados pelo programa informático Alceste $4.8^{11}$ a partir da classificação hierárquica descendente - CHD, que, utilizando as UCEs com tamanhos diferentes, deu origem a duas classes distintas ou categorias. Os resultados empíricos foram interpretados a partir do referencial teórico das representações sociais. ${ }^{9}$

\section{RESULTADOS}

Apresentaremos, portanto, o breve perfil dos sujeitos participantes do estudo e, em seguida, as representações sociais sobre violência em idosos, atendendo aos objetivos propostos neste estudo.

Tabela 1. Perfil dos idosos avaliados $(n=30)$.

João pessoa/PB, 2009.

\begin{tabular}{rrr}
\hline \multicolumn{3}{c}{ PROFISSIONAIS DO ESTUDO } \\
\hline Idade & $\boldsymbol{n}$ & $\%$ \\
$20-30$ & 02 & $50 \%$ \\
$31-40$ & 01 & $25 \%$ \\
$41-50$ & 01 & $25 \%$ \\
Sexo & $\boldsymbol{n}$ & $\%$ \\
Masculino & 01 & $25 \%$ \\
Feminino & 03 & $75 \%$ \\
\hline
\end{tabular}

De acordo com a Tabela 1, há uma prevalência de idosos na faixa etária 60 a 65 anos, com $37 \%$ e entre 71 a 75 anos correspondendo a $30 \%$ dos idosos entrevistados, com predomínio para o sexo masculino.
Quanto aos quatro funcionários avaliados que atendem junto à curadoria do idoso, como pode ser identificado na Tabela 2, 50\% profissionais estão na faixa etária de 20 a 30 anos, e $50 \%$ nas demais faixas. Dos quatro funcionários, $75 \%$ são do sexo feminino e $25 \%$ são do sexo masculino.

Tabela2. Perfil dos profissionais da curadoria do cidadão/ delegacia especializada avaliados ( $n=4)$. João Pessoa/ $\mathrm{PB}, 2009$.

\begin{tabular}{rcc}
\hline \multicolumn{3}{c}{ IDOSOS DO ESTUDO } \\
\hline Idade & $n$ & $\%$ \\
\hline $60-65$ & 11 & $37 \%$ \\
$66-70$ & 06 & $20 \%$ \\
$71-75$ & 09 & $30 \%$ \\
$76-80$ & 04 & $13 \%$ \\
\hline Sexo & $n$ & $\%$ \\
\hline Masculino & 16 & $53 \%$ \\
\hline Feminino & 14 & $47 \%$ \\
\hline
\end{tabular}




\section{CONTEXTOS SEMÂNTICOS SOBRE A VIOLÊNCIA EM IDOSOS}

Neste estudo descreveremos o resultado obtido do processamento dos dados realizado pelo programa informático software Alceste 4.8 fazendo inferências e interpretações dos resultados mais significativos do corpus pesquisado. Os dados submetidos constituído por 34 textos ou Unidades de Contexto Iniciais (UCl's), por meio de uma análise hierárquica descendente, resultaram em 105 palavras, o equivalente a um aproveitamento do corpus de $86,87 \%$. A análise do material foi baseada na elaboração de segmentos cognitivos construídos pelos idosos e profissionais, apontados nas classes delimitadas, através do programa informático adotado. De acordo com esta orientação foram identificadas duas classes de segmentos (UCE's) de texto ou categorias temáticas, diferentes entre si, a saber: sentidos da violência para os profissionais do serviço de curadoria do cidadão/delegacia especializada; e sentido da violência para os idosos, apresentadas a seguir.

Classe 1. Sentidos da Violência para os Profissionais do Serviço de Curadoria do Cidadão/Delegacia Especializada.

A classe um detém 50 UCE's, o que corresponde a $58,14 \%$ das UCE's retidas. Percebemos que, mesmo sendo o número de profissionais menor, esta classe foi a maior por ser os profissionais os depoentes que mais se expressaram sobre a temática em estudo. Nela foram selecionadas 45 palavras, exemplificadas a seguir aquelas com maior frequência ou quiquadrado, conforme Tabela 3.

Tabela 3: Palavras associadas significativamente à classe

1. João Pessoa/PB, 2009.

\begin{tabular}{lcc}
\hline \multicolumn{1}{c}{ Palavras } & Frequência & $\lambda^{2}$ \\
\hline Agredido & 08 & 06.35 \\
Agressão & 10 & 08.15 \\
Amigos & 24 & 23.97 \\
A to & 07 & 02.38 \\
Briga & 09 & 07.24 \\
Desrespeito & 08 & 06.35 \\
Dor & 25 & 12.91 \\
Física & 17 & 07.89 \\
Injustiça & 06 & 04.64 \\
Maltratado & 08 & 06.35 \\
Medo & 15 & 09.25 \\
Pessoa & 13 & 04.41 \\
Raiva & 15 & 13.08 \\
Responder & 20 & 10.87 \\
Sofrimento & 06 & 04.64 \\
Violência & 81 & 07.37 \\
\hline
\end{tabular}

Os sujeitos do estudo, profissionais do serviço de curadoria do cidadão/delegacia especializada, consideram a "violência", ser "agredido" ou qualquer forma de agressão descrita como um "ato» que decorre de uma «briga», "raiva»; é o idoso ser "maltratado", capaz de causar "sofrimento", "raiva", "injustiça», "desrespeito" e "dor" quer "física" ou moral, levando a "pessoan idosa a ter "medo».

A concepção de violência tanto por parte dos profissionais quanto dos próprios idosos não é muito diferente do que se identifica em outras épocas acerca do lado silencioso de um comportamento que é tão antigo quanto a própria humanidade. É engano pensarmos nos problemas sociais como se fossem algo novo, acreditando tratar-se de um tema que 0 mundo nunca conheceu. Tais pensamentos vão desde os maustratos à violência em si, uma vez que não é um fenômeno novo. A agressão e a violência para com os pais foram temas de lendas e de literatura durante milhares de anos; a mitologia grega está repleta de histórias de parricídio, ressaltados na atualidade por romancistas e dramaturgos apontando esses temas de conflitos na família, quando os pais atingem a velhice. Sabemos que o avanç̧o das leis de proteção para a pessoa idosa e com práticas de cidadania mais efetiva a violência vem deixando de ser um tema velado para ser tratado do ponto de vista humanitário, ou melhor, jurídico. A história da família revela 
igualmente que conflitos com os pais, por vezes com consequências trágicas, não é um fenômeno pouco comum como atesta os achados evidenciados nesse estudo e em muitos outros, tanto no contexto nacional quanto internacional. ${ }^{8,12}$

Esta preocupação de origem americana é justificada baseada em cinco fatores diante dos maus-tratos aos idosos, como: o aumento da população idosa, maior atenção a outras formas de violência na família, uma legislação que foi aprovada nos Estados Unidos e em demais países, uma atividade ao nível local e uma intensa publicidade nos meios de comunicação. ${ }^{12}$

Nesta classe, os diferentes sentidos atribuídos pelos profissionais à violência retratam as diferentes representações sociais expressas a partir de suas experiências cotidianas enquanto profissionais que atendem idosos vítimas de violência. Estas dimensões são exemplificadas nas UCE's desta classe, conforme exemplos de segmentos de falas de profissionais apresentados a seguir:

[...] dor, ferida, raiva, agressão, soco [...] um ato desumano [...] agressão física, dor, autoritarismo, humilhação, medo [...] briga entre duas ou mais pessoas [...] vontade de justiça, humilhação [...] falta de amor no coração das pessoas [...] agressão física, sofrer violência é uma falta de respeito [...] uso excessivo de força, intimidação moral, crueldade, constrangimento [...] dependência, desrespeito a minha idade, guerra, brigas [...] sangue, dor de todas as formas, agressão moral [...] (profissionais)

Sabe-se que diversas formas de violência configuram-se atualmente um sério problema de saúde pública no Brasil e no mundo, por se apresentar multifacetado podendo ser percebido nos diferentes espaços, quer públicos ou privados; embora a mídia venha dando destaque no dia-a-dia, a violência ainda é frequentemente abordada como um assunto restrito à segurança pública. ${ }^{12,13}$ Logo, qualquer que seja a forma de violência utilizada, todos os tipos de maus-tratos aos idosos implicam, fundamentalmente, uma violação de confiança, em que a saúde mental ou física de uma pessoa idosa é afetada por outra pessoa queé responsável pelo seu bem-estar. Esses maus-tratos vão desde a violência física, que inclui espancamentos, bofetadas, pontapés, abanões ou outras formas explícitas de força física para magoar, à negligência intencional ou não, maus-tratos psicológicos e os relacionados à exploração financeira.

\section{Classe 2. Sentidos da Violência para os Idosos.}

A classe dois detém 36 UCE's, o que corresponde a 41,86 \% das UCE's retidas, com 17 palavras selecionadas com maior frequência, ou qui-quadrado, com um maior conteúdo apesar de ter um número maior de sujeitos da pesquisa, mas ainda com dificuldades em falar sobre o tema, quer pelas condições psicológicas ou por questões de ordem moral ou medo, apresentadas na Tabela 4.

Tabela 4. Palavras associadas significativamente à classe 2. João Pessoa/PB, 2009

\begin{tabular}{lcc}
\hline Palavra & Frequência & $\boldsymbol{\lambda}^{2}$ \\
\hline Vida & 16 & 08.87 \\
Triste & 09 & 13.96 \\
Consequência & 29 & 60.77 \\
Filho & 04 & 05.83 \\
Impotente & 08 & 03.98 \\
Situação & 10 & 06.76 \\
Sofrido & 18 & 31.62 \\
\hline
\end{tabular}

A classe dois: os sentidos da violência para o idoso contempla os conteúdos semânticos em que os sujeitos fazem alusão à violência como uma "situação" que acarreta na "vida" do idoso "consequência". 0 idoso fica "triste" por ser muitas vezes ser agredido pelo "filho", o que o deixa "impotente" $e$ "sofrido". Tais representações podem ser observadas nos exemplos abaixo:

[...] meu filho deveria cuidar de mim nessa fase da minha vida, é muito triste passar por isso. [...] quando sofri violência me senti impotente [...] ter sofrido violência e uma vida com limites [...] sofrido violência e uma vida de preocupação
[...] minha situação ao sofrer violência é viver o dia a dia com desanimo, triste [...] sentir-se impotente [...] uma má qualidade de vida, é ser maltratada diariamente pela pessoa que trabalha comigo e me sinto indefesa [...] uma vida mais curta, minha situação ao sofrer violência e me sentir impotente por ser idoso [...] sofrer violência considero uma vida triste [...] viver triste com a situação que estou vivendo [...] não realizar minhas necessidades básicas, como saúde [...] passo por tudo isso porque dependo do meu filho para realizar as atividades da vida diária [...] (Idosos). 
Estas representações deixam evidente o cenário de diferentes práticas de violências responsabilizando as relações familiares, dentro de suas casas, sendo os autores, em sua maioria, os membros da família. Observa-se nessas falas que a violência física, por exemplo, é frequentemente acompanhada de violência psicológica e exploração financeira, às vezes acompanhada de negligência. Estes resultados, em aparente identificação, são semelhantes aos encontrados em estudos realizados em diferentes países e culturas, ${ }^{12}$ sugerindo uma sociedade na qual a expectativa de vida está se expandindo, criando situações inovadoras, sem que haja maior preparação de seus membros para lidarem com esta nova situação. ${ }^{14}$

Diante de tal situação, pode-se inferir a responsabilidade da família no abandono dos idosos. A própria família é responsável por diferentes tipos de violência e, ao invés de serem protegidos por terem passado toda sua vida cuidando de filhos e familiares, os idosos são vítimas de diferentes formas de violências, corroborando a triste realidade do mundo capitalista, onde não há mais quem fique em casa cuidando daquele que envelheceu; há a necessidade de se buscar uma alternativa viável, daí a institucionalização. ${ }^{15}$ Muitas vezes a visão negativa que o idoso tem de si próprio decorre da sua significação perante a sociedade e o papel que ele desempenha perante a sua família., 16,17

Esta realidade social apreendida a partir das representações sociais construídas por idosos e profissionais retrata uma situação em que o idoso ainda é visto com muitas restrições e olhares preconceituosos, na qual a velhice é sinônimo de senilidade e predominam diferentes mitos: velhice como impotência, doença, alienação, inflexibilidade, entre outros.

Assim, é cada vez mais comum se observar na pessoa idosa um comportamento de fechamento e afastamento das outras, ano após ano, como uma espécie de cerca imaginária cada vez mais alta, entre ele e os outros, às vezes como uma forma de se proteger intimamente dos próprios familiares.

\section{CONSIDERAÇÕES FINAIS}

Este estudo procurou conhecer os sentidos associados à violência pelos idosos e profissionais do serviço de curadoria do cidadão/delegacia especializada que atendem os idosos no momento das suas queixas. Embora os resultados e a análise feita nesse estudo não ofereçam uma perspectiva geral do que vem sendo investigado nessa temática, este recorte sobre representações de violência para idosos e profissionais procura apresentar uma realidade social que não é muito diferente de outras, e daí se pode repensar ações mais efetivas diante da violência dirigida a pessoa idosa.

Dentre algumas sugestões, pode-se inferir que a realização de estudos mais amplos que investiguem melhor os fatores de risco a que estão submetidos os idosos constitui um aspecto de suma importância. Alguns estudos realizados apontam alguns fatores como: condições de habitação (viver só ou a coabitação); isolamento social; demência; problemas de saúde mental e de dependência de substâncias; dependência dos agressores; saúde e capacidades funcionais do idoso; violência nos casais idosos; deteç̧ão; serviços de proteção de adultos, entre outros. ${ }^{12}$ Constatamos a necessidade de pesquisas mais aprofundadas sobre esse tema que possam direcionar ações efetivas para inibir tais práticas, já milenares.

As representações sociais sobre a violência contra a pessoa idosa e o seu impacto para os profissionais diante desta problemática apontam que os estudos até agora realizados no Brasil ainda permanecem na esfera da saúde e atribuem esse fenômeno um problema de saúde pública. Assim sendo, esse estudo oportunizou a investigação de um fenômeno que transcende ao campo da saúde, pelo quadro evidenciado, em que os maus-tratos e a negligência contra os idosos constituem um grave problema, ainda não diagnosticado em toda a sua extensão, sobretudo no âmbito familiar e institucional, merecendo, portanto, maior atenção de órgãos governamentais e não governamentais. A saúde é evidenciada por estarem os profissionais de saúde que prestam assistência ao idoso com maior probabilidade de se depararem com esse problema se considerarem quase um problema rotineiro. Com efeito, os maus tratos a idosos são suficientemente frequentes para serem encontrados diariamente e com regularidade na prática clinica. ${ }^{12}$

Desta forma, assim como os serviços, os profissionais de saúde parecem ser os mais envolvidos com a ocorrência de violência. Logo, esta situação exige um preparo dos diferentes profissionais tanto da atenção primária de saúde quanto dos profissionais da área social e do direito, para identificação, prevenção e intervenção em casos de maus-tratos e negligência à pessoa idosa.

\section{REFERÊNCIAS}

1. Chamoivicz FA. A saúde dos idosos brasileiros às vésperas do século XXl: problemas, projeções e alternativas. Rev Saude Publica. 1997; 31(2): 184-200.

2. Marziale MHP. A política nacional de atenção ao idoso e a capacitação dos profissionais de enfermagem. Rev Latino-am Enfermagem. 2003; 11(6): 701-02.

3. Fonseca RP, Trentini CM, Valli F, Silva, RAN. Representações do envelhecimento em agentes comunitários da saúde e profissionais da enfermagem comunitária: aspectos psicológicos do processo saúdedoença. Cien Saude Colet. 2008 jul/ago; 13 (4): 1275-84.

4. Prochet TC, Silva MJP. Situações de desconfor to vivenciadas pelo idoso hospitalizado com a invasão do espaço pessoal e territorial. Esc Anna Nery 2008 jun; 12 (2): 310-15. 
5. World Health Organization -WHO. The Toronto Declaration on the Global Prevention of Elder Abuse. Geneva; 2002.

6. Vieira LES, Arcoverde MLV, Araújo MAL, Ferreira RC, Fialho AVM, Pordeus ANJ. Impacto da violência na saúde de famílias em Fortaleza, Ceará. Cien Saude Colet. 2009 nov/dez; 14 (5): 1773-79.

7.Ministério da Saúde(BR). Estatuto do idoso. [Internet]. [citado 2008 set 12]. Disponível em: http://www.serasa.com.br/guiaidoso/20.htm.

8. Minayo MCS, Coimbra Júnior CEA. Antropologia, saúde e envelhecimento. Rio de Janeiro (RJ): Fiocruz; 2004.

9. Moscovici S. A representação social da psicanálise. Tradução Álvaro Cabral. Rio de Janeiro (RJ): Zahar; 1978.

10. Jodelet $D$. Representações sociais: um domínio em expansão. In: As representações sociais. Rio de Janeiro (RJ): UERJ; 2001. p. 17-44.

11. Moreira ASP.Perspectivas teórico-metodológicas em representações sociais. João Pessoa (PB): Universitária /UFPB; 2005.

12. Fundação Calouste Gulbenkian. Fórum Gulbenkian de Saúde sobre o envelhecimento. 0 tempo da vida.. Principia (PT): Cascais; 2009.

13.Ministério da Saúde (BR). Conselho Nacional das Secretarias Municipais de Saúde - CONASS. 0 desafio do enfrentamento da violência: situação atual, estratégias e propostas. Brasília (DF); 2008.

14. Alcântara AO. Velhos institucionalizados e família: entre abafos e desabafos. Campinas (SP). Alínea; 2004.

15. Chaimowicz F, Greco DB. Dinâmica da institucionalização de idosos em Belo Horizonte, Brasil. Rev Saude Publica. 1999 out; 33(5): 454-60.

16. Zimerman Gl. Velhice: aspectos biopsicossociais. Porto Alegre (RS): Artmed; 2000.

17. Lemos MTTB, Zagaglia RA. A arte de envelhecer: saúde, trabalho, afetividade, estatuto do idoso. Rio de Janeiro (RJ): UERJ; 2004. 